\title{
In case you missed it: Highlights of the Congenital Cardiac Sessions at The American Association for Thoracic Surgery 95th Annual Meeting of 2015
}

\author{
Marshall L. Jacobs, MD
}

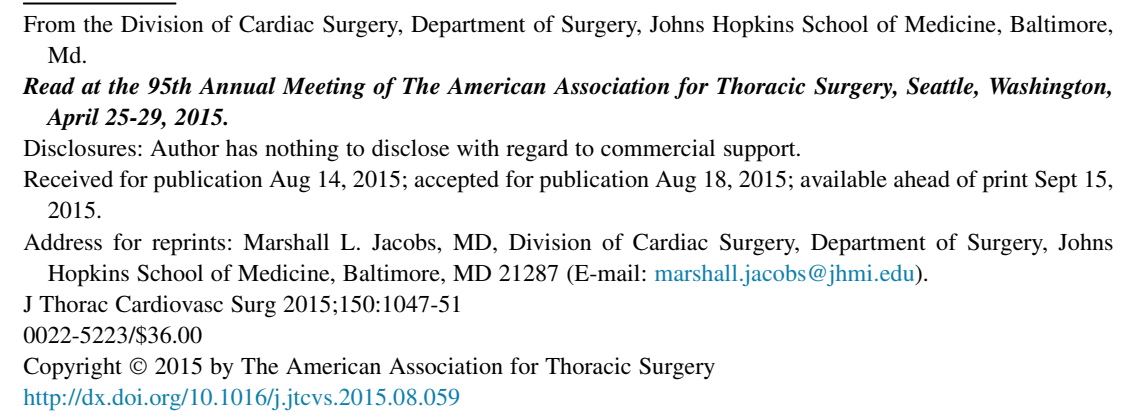

"In Case You Missed It" (ICYMI) is the name given to a series of sessions held on the morning of the final day of the 95th Annual Meeting of The American Association for Thoracic Surgery 2015. The overall program included so many high points of special interest to those involved in surgery for congenital heart disease that it seems appropriate to acknowledge that each meeting attendee can only be in one place at one time, creating the likelihood that most people missed something that would be of interest to them. In addition, for those who were not able to be in Seattle in April, this brief account of the congenital heart disease ICYMI session held on April 29, 2015, provides a convenient summary of some of the important presentations throughout the meeting.

In the opening plenary session, Dr Travis Wilder, the Congenital Heart Surgeons' Society Kirklin-Ashburn fellow, presented a study that was based on an inception cohort of neonates with critical left ventricular outflow tract obstruction of whom 454 (of the larger total cohort) had embarked on a pathway beginning with a Norwood-type palliation as the first surgical step. The intent was to look at such events as mortality, but importantly also at ventricular function and tricuspid regurgitation, as time-related phenomena, comparing those who underwent a Norwood procedure with a Blalock-Taussig shunt with those who underwent a right ventricle-to-pulmonary artery conduit (RV-PA; Sano modification). Vital status over time differed between the groups, and in an unadjusted analysis the survival at all points in time was better among those in the RV-PA group. With the goal of looking more closely at other measures, a propensity scoring system was used to compose groups that were relatively comparable with respect to most baseline conditions, specifically things that are generally acknowledged as risks factors for this type of surgical palliation. And about three-quarters of the entire cohort were able to

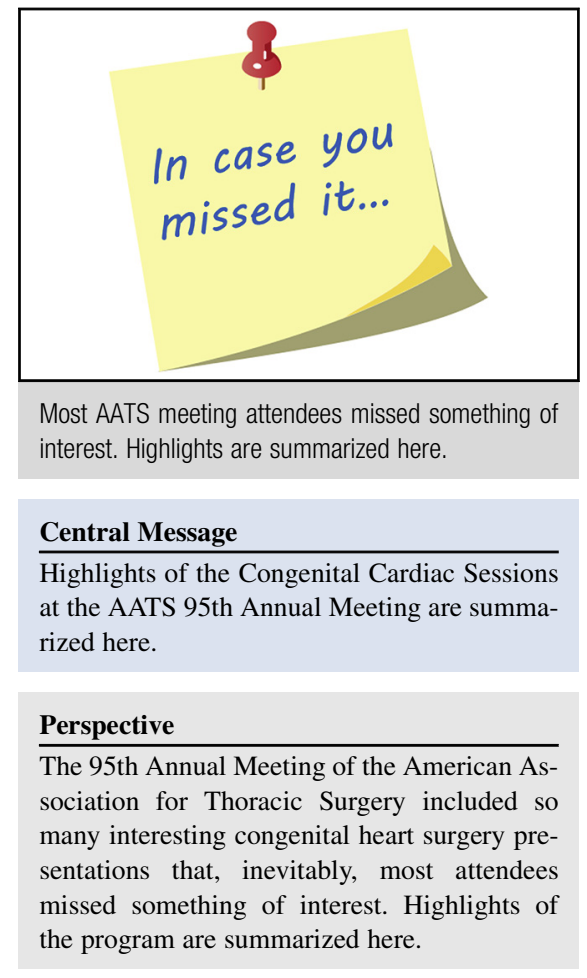

be paired with the propensity scoring system, enabling an "apples to apples" comparison. For the 338 patients (of the entire cohort of 454) who constituted these matched pairs, there still appeared to be a survival advantage at all points in time associated with the RV-PA conduit strategy (Figure 1).

Looking further, data from nearly 3000 echocardiograms performed during the entire period of follow-up was used to classify each patient in the propensity-matched groups at each point in time with respect to having either important right ventricular dysfunction or tricuspid regurgitation (graded as moderate or greater). There was an early prevalence of important right ventricular dysfunction that peaked at about $12 \%$ for the entire cohort. When stratified by type of palliation, that prevalence was greater among the Blalock-Taussig shunt group than among the RV-PA group. Getting out beyond that early peak, however, the prevalence of moderate or greater right ventricular dysfunction was actually very low and relatively constant in both groups. An important observation here was the similarity in the time course between the hazard function for mortality and 


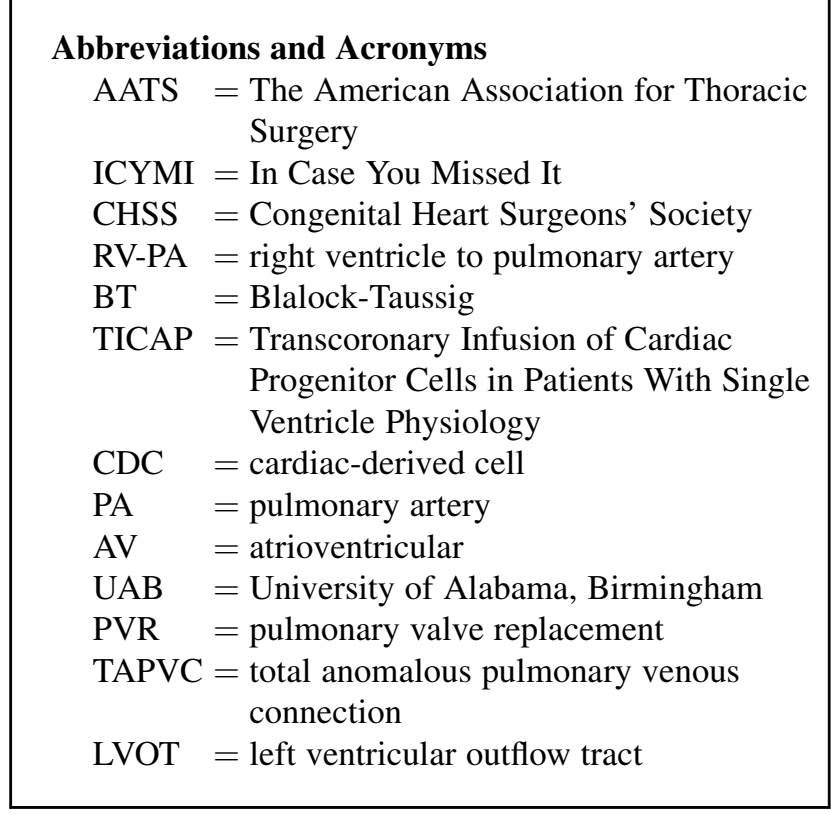

the prevalence of right ventricular dysfunction. Further analysis is going to have to involve a deeper look at these relationships, because with the observed similarity in chronicity between right ventricular dysfunction and mortality, one cannot really dissect out how much of the low, steady-state prevalence of right ventricular dysfunction beyond the early period is related to patients with right ventricular dysfunction dying early and leaving the series, as opposed to how much it is related to improvement in right ventricular function related to the unloading strategy. It eventually may be possible to determine whether late trends in right ventricular function are associated with the initial palliative strategy. The methodology used in this study represents a new way of looking at the relationship between right ventricular dysfunction and mortality, and time will tell whether the observed survival advantage with the RVPA conduit strategy will persist through a longer period of follow-up.

In the same opening plenary session, Dr Shunji Sano, on behalf of his colleagues at Okayama University, presented a 3-year follow-up status report on the Transcoronary Infusion of Cardiac Progenitor Cells in Patients With Single Ventricle Physiology (TICAP) trial, which explored the transcoronary infusion of cardiac progenitor cells in patients with hypoplastic left heart syndrome. This phase I trial was predicated on the observations that there seems to be a large population of cardiac stem cells in very young children and that these "young stem cells" may have greater regenerative potential than older cells in older subjects. This was a phase I study, with the principle objectives of proof of concept and demonstration of feasibility and safety. Eighteen patients were enrolled, beginning in 2011, and ultimately 7 patients who received cardiac-derived stem cells were compared

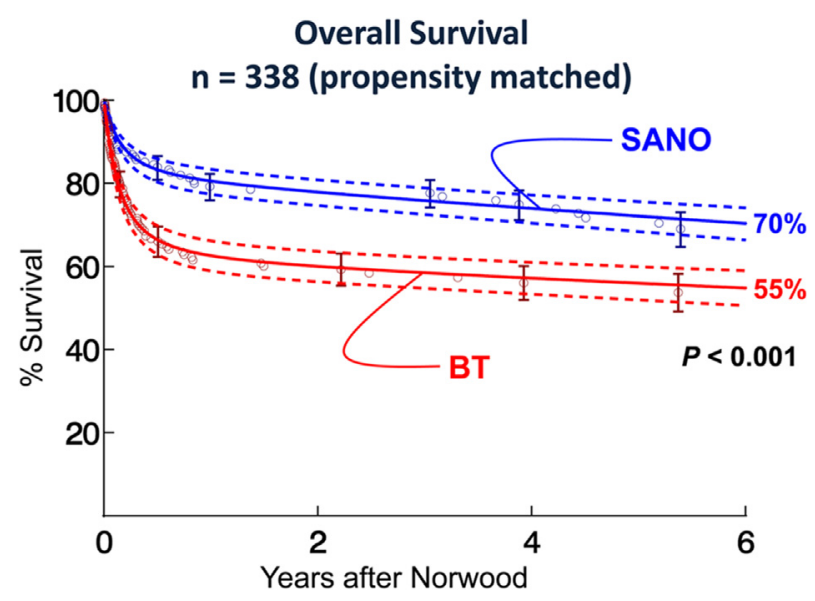

FIGURE 1. For the 338 propensity-matched patients (169 matched pairs, of the entire cohort of 454), there appeared to be a survival advantage at all points in time associated with the right ventricle-to-pulmonary artery conduit strategy $(S A N O)$ in comparison with the modified BlalockTaussig shunt strategy (BT). Adapted from the presentation by Dr Travis Wilder.

with 7 control patients. In this phase I trial, there was no randomization, and there were some issues with matching between groups. In the cardiac derived cell-treated group, the subjects overall were slightly older than those in the control group. Importantly, the timing of the primary study intervention, the stage during the course of palliative reconstruction at which the study intervention occurred, differed somewhat between the groups. That having been said, there was a striking absence of adverse events in both groups, which is of fundamental importance in a feasibility and safety trial. There was no evidence of myocardial ischemia, ventricular arrhythmia, or infection throughout the 36-month period. An observed trend within the treated group toward improved hemodynamic parameters, such as right ventricular ejection fraction, may justify optimism regarding efficacy. I believe, however, that it is prudent to await the results of the planned prospective, randomized phase II trial before drawing conclusions about potential hemodynamic benefits. There were enough differences between groups in the phase I study that one really can only draw conclusions regarding feasibility and safety while awaiting data on efficacy from future studies.

Of several conclusions presented by Dr Sano, I think that the most important is that intracoronary infusion of autologous cardiac-derived stem cells appears to be feasible and safe. The efficacy of this strategy is going to be explored by this group of investigators in a phase II trial. Additional trials are underway elsewhere. A study being done at the Mayo Clinic differs from the Okayama study in that it uses autologous cord blood-derived stem cells, and a trial is being begun by Dr Sunjay Kaushal and associates in Baltimore with mesenchymal homologous stem cells. 
Also in the first plenary sessions was a cohort study of patients who had undergone Ross operations at Boston Children's Hospital. Their experience with the Ross operation is large and has been very encouraging, as it has been at so many places. In this study, the researchers looked in particular at a smaller group of patients who had previously undergone univentricular palliation because of hypoplasia of left heart structures and then ultimately underwent conversion to biventricular circulation with takedown of the aortopulmonary amalgamation. Biventricular repair included pulmonary autograft reconstruction of the left ventriculoaortic junction. Not surprisingly, these patients undergoing the "complex Ross operation" in the course of conversion to biventricular circulation were younger than the remainder of the Ross cohort, and their follow-up was somewhat shorter at a median of 18 months as opposed to 42 months. Patient survival, which was $100 \%$ in the simple Ross population, was also very encouraging among the complex biventricular conversion Ross population. There were 2 deaths among the 12 patients, neither of which death was related to the autograft itself.

Freedom from neoaortic valve reintervention likewise was very encouraging and was not statistically different between the simple and complex Ross groups (Figure 2). Importantly, the degree of aortic regurgitation at late follow-up appeared to be unrelated to the initial pulmonary valve $z$ score, so that these patients with relatively tiny native left ventricular outflow tracts who underwent autograft implantation of the relatively large pulmonary valve and trunk into the left ventricular outflow tract did not appear to have a higher risk of autograft regurgitation than that of the comparison group. Their conclusion was that the Ross operation can be considered for patients with significant aortic valve disease, left ventricular outflow tract hypoplasia, or both undergoing conversion to a biventricular circulation after univentricular palliation.

The remaining sessions included several presentations looking at late events after the Fontan operation. In the first of these studies, investigators from the Children's Hospital of Philadelphia reviewed a cohort of 773 patients who underwent Fontan operations from 1992 to 2009. Nearly two-thirds of the cohort had right ventricular dominant morphology. and fully one-half of the cohort had hypoplastic left heart syndrome. Roughly half of the cohort had a lateral tunnel, and half had an extracardiac conduit. Fenestrated Fontan procedures were done in $90 \%$ of cases. By means of the Death Master File, they were able to ascertain virtually complete data on the vital status of this entire cohort with a median follow-up of 9 years, with onefourth of the patients having follow-up beyond 15 years. There were 79 deaths during the study period, approximately half of which were in the first year after the Fontan procedure. Kaplan-Meier analysis of overall survival

\section{Freedom from Neo-aortic Valve Re-intervention}



FIGURE 2. When comparing the simple and complex Ross operation groups with respect to neoaortic (neo-Ao) valve reintervention, there were no significant differences between groups. $I Q R$, Interquartile range. Adapted from the presentation by Dr Alejandra Bueno.

revealed 2 noteworthy findings. The first was an early hazard phase for mortality, in the first year after the Fontan operation. The second was the encouraging survival of approximately $80 \%$ at 20 years after the Fontan operation. When this survival was stratified by era of surgery, it was apparent that there is an era effect, but also that almost all of that era effect was related to the very early operative mortality in the earliest era. On examination of late survival conditional on survival to 1 year, the era effect was ablated. A risk factor analysis was done to look at factors associated with mortality in the first year after Fontan completion. Prolonged pleural drainage at the time of surgery and earlier era were both associated with risk of mortality in the first year after the Fontan operation, as was pre-Fontan pulmonary arterial pressure greater than $15 \mathrm{~mm} \mathrm{Hg}$ (among those who had catheterization data). Risk factors for late mortality included intensive care unit stay longer than 1 week, common atrioventricular valve, and atrioventricular valve regurgitation before the Fontan procedure. Among those patients who had hemodynamic data available before the Fontan operation, pulmonary arterial pressure greater than $15 \mathrm{~mm} \mathrm{Hg}$ was a risk factor for mortality after the first year. The late survival curves for the lateral tunnel and the extracardiac types of Fontan procedure were virtually superimposable (Figure 3). In addition, the late survivals of patients with dominant right and left ventricular morphologies were also indistinguishable from a statistical standpoint, with no apparent survival disadvantage for those with right ventricular morphology.

Another very interesting perspective on late events after the Fontan operation was presented by Dr Thomas Carins, on behalf of investigators from Royal Children's Hospital, Melbourne, Australia. This is one of many studies based on a collaborative New Zealand-Australia Fontan Registry that includes data on well over a thousand patients. With 


\section{Late Survival by Fontan Type (conditional on survival to 1 year)}

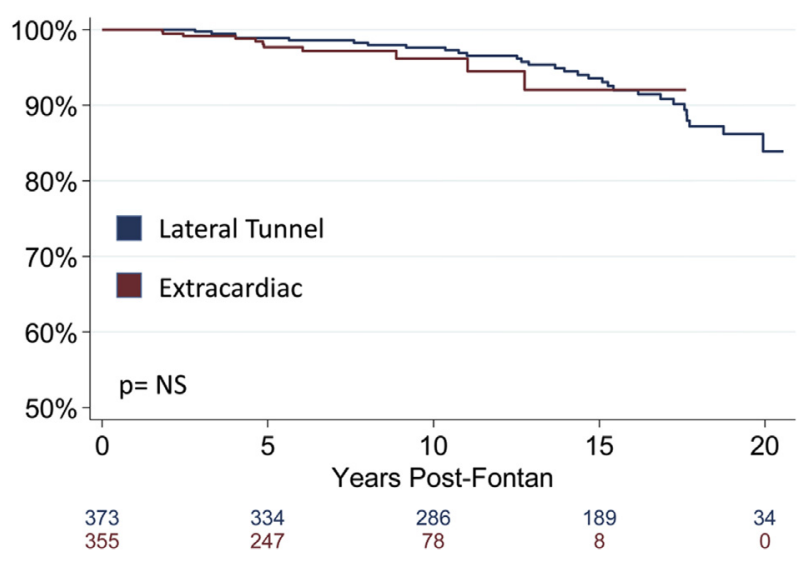

FIGURE 3. Examining late survival conditional on survival to 1 year, the late survival curves for the lateral tunnel and the extracardiac types of Fontan procedure were virtually superimposable. NS, Not significant. Adapted from the presentation by Dr Tacy Downing.

1272 enrolled patients, cross-sectional analysis performed in the last year identified 210 patients surviving with a Fontan circulation who have had arrhythmias. In this study, every one of those cases was reviewed in detail-including 174 cases of tachyarrhythmia and 80 cases of bradyarrhythmia. At 20 years, freedom from tachyarrhythmia was $58 \%$ and freedom from bradyarrhythmia was $86 \%$. Not surprisingly, freedom from tachyarrhythmia was lower in the group that had undergone aortopulmonary connection, whereas this did not appear to be a differentiating factor with regard to the freedom from bradyarrhythmia.

The investigators looked at events after the first occurrence of arrhythmia. They observed that there was a steady gradual attrition after the first onset of arrhythmia, with survival of $72 \%$ of patients at 20 years after the first occurrence of tachyarrhythmia. Interestingly, the survival after occurrence of arrhythmia did not appear to be different between types of Fontan connections. Among patients with all types of Fontan connections, however, there appeared to be the initiation of a trend toward progressive decline in ventricular function, which seemed to coincide temporally with the onset of arrhythmia. Whether the arrhythmia is a marker of this decline or whether it is a contributing factor remains to be seen, but there was a trend suggesting progressive decline in ventricular function and occurrence of other adverse events after the onset of arrhythmias in patients with Fontan circulations.

Another interesting look at the Fontan population during the long term explored a very different phenomenon. Health-related quality of life was the focus of the study presented by Dr James Kirklin on behalf of his colleagues at University of Alabama, Birmingham. Importantly, the overall survival was quite similar to that reported by the group from Children's Hospital of Philadelphia. At University of Alabama, Birmingham, survival was about $80 \%$ at 20 years. And the important finding in this study, which was based on patient-provided information with an SF-36 health survey, is that on average the scores of patients with Fontan circulations were virtually the same as population norms for mental capacity domains, although they were lower for physical capacity domains. And in a comparison of these metrics between the patients with Fontan circulations and a contemporaneous group of patients who had undergone heart transplants, there were no differences between the groups.

During the next plenary session, investigators from Boston examined the long-term outcomes after pulmonary valve replacement. They retrospectively reviewed 633 patients who underwent pulmonary valve replacement (PVR) with bioprosthetic valves at Children's Hospital Boston from January 1996 to February 2014. They observed that among patients younger than 12 years at the time of PVR, those younger than 6 years and those aged 6 to 12 years at the time of PVR had similar time-related freedoms from reintervention. Patients who were 12 years old or older at the time of PVR had greater time-related freedom from reintervention, with little difference between those aged 12 to 18 years and those who were 18 and older. Very importantly, however, after adjustment for age, the time-related risk of reintervention appeared to be higher among those who received the Mitroflow prosthesis (SORIN SpA, Milan, Italy), regardless of age.

Dr Bahaaldin Alsoufi and colleagues at Emory University summarized their experience with repair of interrupted aortic arch and ventricular septal defect. As at many centers, their approach was adapted to the individual patient, with most of the cohort undergoing a standard repair (direct anastomosis with patch augmentation) but those considered to be at higher risk for left ventricular outflow tract obstruction undergoing either conal resection or bypass of the left ventricular outflow tract by means of a modified Yasui or a Norwood procedure followed by an eventual Norwood-Rastelli type of reconstruction. As in reports on the Congenital Heart Surgeons' Society multi-institutional cohort, the Emory group observed a steady increase over time in reoperations on the left ventricular outflow tract (LVOT). Notably, freedom from reoperation on the LVOT for patients who underwent conal resection at the time of initial repair was considerably less than it was among those who had undergone an aortopulmonary amalgamation to bypass the hypoplastic LVOT, which was similar to those with minimal LVOT hypoplasia who had undergone a standard repair (Figure 4).

Finally, there were 3 presentations that provided information on the very challenging problem of pulmonary venous disease. A study from the Guangdong Cardiovascular Institute in China, presented by Dr Yiqun Ding, compared 




FIGURE 4. Time-related freedom from reoperation on the left ventricular outflow tract (LVOT) for patients who underwent conal resection at the time of initial repair was considerably less than it was among those who had undergone an aortopulmonary amalgamation to bypass the hypoplastic left ventricular outflow tract. Adapted from the presentation by Dr Bahaaldin Alsoufi.

nonrandomized cohorts who underwent either conventional repair or sutureless repair of total anomalous pulmonary venous connection. Their observation was that among patients who preoperatively had obstructed pulmonary venous return, the mortality was substantially higher among those who had undergone a conventional repair than among those who had undergone a sutureless repair. As at several centers that have adopted sutureless repair as the primary approach to management of total anomalous pulmonary venous connection, these investigators had enthusiasm for sutureless repair as a primary approach and were encouraged by their results. The comparison between techniques, however, was tempered by issues regarding differences between the treatment groups and the fact that different surgeons had used the conventional and the sutureless techniques.

Dr Christopher Baird from Boston talked about management strategy and outcomes for the enormously challenging group of patients with primary or native pulmonary vein stenosis, illustrating the important anatomic compressive features that are a less widely appreciated aspect of this disease. He explained the interaction between right pulmonary artery and right upper pulmonary vein, the effect of mediastinal shift, and even the effect of loss of pulmonary volume that may contribute to the potential compression of pulmonary veins by contiguous structures. Although it is acknowledged that native pulmonary vein stenosis is a complicated, incompletely understood endothelial proliferative disease, it is nonetheless important to recognize anatomic features that may be addressed at the time of surgery.

Dr Mauro Lo Rito from the Hospital for Sick Children, Toronto, Ontario, Canada, introduced a scoring system to look at the downstream and upstream dimensions in stenotic pulmonary veins. This system of measurement has the potential to help with stratification of subjects and assessment of substrate and effect in clinical trials designed to evaluate various strategies to address intrinsic obstructive disease of the pulmonary veins.

There were so many more presentations of interest to congenital heart surgeons at the 2015 annual meeting of the American Association for Thoracic Surgery that it would be impractical to review all of them. Those that are summarized here are among those that addressed unresolved challenges that are faced by those dealing with complex congenital heart disease. There can be little doubt that we will hear more about these same problems at future American Association for Thoracic Surgery meetings.

Key Words: congenital heart disease, congenital heart surgery, professional affairs 